\title{
Amélioration des conditions d'hygiène et d'assainissement dans la commune de Zè au Bénin
}

\section{Roch Christian Johnson}

Université d'Abomey-Calavi, Centre Interfacultaire de Formation et de Recherche en Environnement pour le Développement Durable (CIFRED),

Laboratoire d'Hygiène, d'Assainissement, de Toxicologie et de Santé Environnementale (HECOTES), Cotonou, Bénin

\section{Armelle Sabine Yélignan Hounkpatin}

Université d'Abomey-Calavi, Centre Interfacultaire de Formation et de Recherche en Environnement pour le Développement Durable (CIFRED),

Laboratoire d'Hygiène, d'Assainissement, de Toxicologie et de Santé Environnementale (HECOTES), Cotonou, Bénin

Université Nationale des Sciences, des Technologies, de l'Ingénierie et des Mathématiques (UNSTIM), Ecole Normale Supérieure de l'Enseignants Technique (ENSET), Lokossa, Bénin

\section{Francine Innocentia Johnson Gratien Boni}

Université d'Abomey-Calavi, Centre Interfacultaire de Formation et de Recherche en Environnement pour le Développement Durable (CIFRED),

Laboratoire d'Hygiène, d'Assainissement, de Toxicologie et de Santé Environnementale (HECOTES), Cotonou, Bénin

\section{Beatriz Gomez. \\ Gabriel Diez.}

Fondation Anesvad, Bilbao Espagne,

General Concha, 28 - $1^{\circ}$ Entrada calle Egaña. 48010 Bilbao, Spain

Doi:10.19044/esj.2021.v17n17p189

Submitted: 12 October 2020

Accepted: 11 May 2021

Published: 31 May 2021
Copyright 2021 Author(s)

Under Creative Commons BY-NC-ND

4.0 OPEN ACCESS

Cite As:

Johnson R.C., Yélignan Hounkpatin A.S., Johnson F.I., Boni G., Gomez B. \& Diez G. (2021). Amélioration des conditions d'hygiène et d'assainissement dans la commune de Zè au Bénin. European Scientific Journal, ESJ, 17(17), 189. https://doi.org/10.19044/esj.2021.v17n17p189 


\section{Résumé}

La présente étude vise à évaluer les effets des interventions mises en œuvre de 2017 à 2018 relatives à la promotion de l'hygiène et de l'assainissement dans la commune de Zè. Réalisée en Septembre 2019, l'étude a mobilisé la recherche documentaire, l'observation et l'entretien. La collecte a été effectuée dans les arrondissements bénéficiaires des interventions et de celles non bénéficiaires. Les résultats après interventions montrent que la proportion des personnes ayant de bonnes pratiques d'hygiène et d'assainissement est passée de $31,01 \%$ à $73,08 \%$. La proportion des groupements de femmes associés aux activités IEC/WASH est passée de $0 \%$ à $87 \%$. Le taux de morbidité liée aux maladies hydro-fécales a régressé de $1,20 \%$ à $0,78 \%$. La prévalence des géohelminthiases a diminué de $7,10 \%$ à $0,75 \%$. Par ailleurs, l'observation a permis de constater la réduction de la défécation à l'air libre, la réduction de la distance séparant les ménages d'un point d'eau, la diminution de la corvée d'eau pour les femmes et les filles, la réduction de la prévalence des maladies courantes dans les localités bénéficiaires. Malgré les résultats positifs obtenus, il reste des défis à relever pour pérenniser les acquis et passer à l'échelle supérieure notamment l'implication effective des autorités locales dans le suivi des ouvrages d'hygiène et d'assainissement.

Mots clés : Eau, hygiène, assainissement, santé, Zè, Bénin 


\title{
Improvement of Hygiene and Sanitation Conditions in the Commune of Zè in Benin
}

\author{
Roch Christian Johnson \\ Université d'Abomey-Calavi, Centre Interfacultaire de Formation et de \\ Recherche en Environnement pour le Développement Durable (CIFRED), \\ Laboratoire d'Hygiène, d'Assainissement, de Toxicologie et de Santé \\ Environnementale (HECOTES), Cotonou, Bénin \\ Armelle Sabine Yélignan Hounkpatin \\ Université d'Abomey-Calavi, Centre Interfacultaire de Formation et de \\ Recherche en Environnement pour le Développement Durable (CIFRED), \\ Laboratoire d'Hygiène, d'Assainissement, de Toxicologie et de Santé \\ Environnementale (HECOTES), Cotonou, Bénin \\ Université Nationale des Sciences, des Technologies, de l'Ingénierie et des \\ Mathématiques (UNSTIM), Ecole Normale Supérieure de l'Enseignants \\ Technique (ENSET), Lokossa, Bénin

\section{Francine Innocentia Johnson Gratien Boni} \\ Université d'Abomey-Calavi, Centre Interfacultaire de Formation et de \\ Recherche en Environnement pour le Développement Durable (CIFRED), \\ Laboratoire d'Hygiène, d'Assainissement, de Toxicologie et de Santé \\ Environnementale (HECOTES), Cotonou, Bénin

\section{Beatriz Gomez \\ Gabriel Diez}

Fondation Anesvad, Bilbao Espagne,

General Concha, 28 - $1^{\circ}$ Entrada calle Egaña. 48010 Bilbao, Spain

\begin{abstract}
The study aims to assess the effects of the interventions implemented from 2017 to 2018 relating to the promotion of hygiene and sanitation in the municipality of Zè. Conducted in September 2019, the study involved documentary research, observation and interview. Collection was carried out in the districts benefiting from the interventions and those not benefiting. Postintervention results show that the proportion of people with good hygiene and sanitation practices increased from $31.01 \%$ to $73.08 \%$. The proportion of women's groups involved in IEC / WASH activities increased from $0 \%$ to $87 \%$. The morbidity rate linked to hydro-fecal diseases fell from $1.20 \%$ to $0.78 \%$. The prevalence of soil-transmitted helminthiasis decreased from $7.10 \%$ to $0.75 \%$. In addition, the observation made it possible to note the reduction in open defecation, the reduction in the distance separating households from a water point, the reduction in the drudgery of water for
\end{abstract}


women and girls, reducing the prevalence of common diseases in beneficiary localities and empowering women in groups through the manufacture and sale of soap.

Keywords: Water, hygiene, sanitation, health, Ze, Benin

\section{Introduction}

L'Eau, l'Hygiène et l'Assainissement (EHA) ont d'importantes répercussions tant sur la santé que sur les maladies (OMS, 2015). En effet, lorsque l'alimentation en EHA est insuffisante, la santé en pâtit considérablement. Aucun autre facteur environnemental déterminant n'a des effets aussi profonds, fragilisant et déshumanisants (Bartram et al., 2010).

Malgré les progrès dans le secteur de l'eau, 785 millions de personnes de la population mondiale boivent de l'eau de source non améliorée sans protection contre la contamination par les matières fécales (McGinnis et al., 2017). Parmi ceux-ci, 159 millions de personnes, dont 58\% vivaient en Afrique subsaharienne et utilisaient les eaux de surface comme eau de boisson (OMS, 2017). Environ deux milliards de personnes dans le monde n'ont pas de toilettes à la maison ou ne disposent pas de toilettes permettant une gestion hygiénique des excréments (McGinnis et al., 2017). En Afrique subsaharienne, environ $31 \%$ des populations sont sans accès à une installation d'assainissement améliorée (OMS, 2017). Cette situation cause chaque année, 1,5 million de décès d'enfants (Awofeso et al., 2013). Les maladies liées à l'insalubrité de l'eau, à l'absence de système d'assainissement et au manque d'hygiène représentent un énorme fardeau pour les pays en développement (McGinnis et al., 2017). Dans ces pays, les écoles en général, et en particulier celles des zones rurales, n'ont généralement pas accès à l'eau potable, à des installations d'assainissement, ni à des dispositifs de lavage des mains. Lorsque ces infrastructures et équipements existent parfois, ils sont souvent insuffisants, tant qualitativement que quantitativement. Les écoles où les conditions d'approvisionnement en EHA sont mauvaises, constituent pour la plupart des environnements à haut risque pour les élèves, le personnel et accroissent la grande vulnérabilité des enfants aux géohelminthiases (Campbell et al., 2014). Aujourd'hui, les Maladies Tropicales Négligées affectent plus d'un milliard de personnes dans le monde et sévissent dans les zones rurales isolées ou les bidonvilles (Ogden et al., 2013).

Au Bénin, la situation des populations en matière d'accès aux systèmes d'assainissement individuel n'est pas reluisante (OMS, 2011). L'enquête démographique et de santé, réalisée en 2017, a montré que 71\% des ménages ont accès à une source d'eau améliorée tandis que $87 \%$ n'ont pas accès à des installations d'hygiène non améliorées. Par ailleurs, parmi les $80 \%$ des personnes disposant d'un endroit pour se laver les mains, (55\%) n'avaient pas 
de l'eau et du savon (INSAE, 2019). Cette situation fait perdre environ 79 millions de dollars US au Bénin chaque année à cause des décès prématurés et 3,1 millions de dollars US du fait des soins de santé dans le cadre du Programme WASH, en 2011.

La population de Zè, située dans le département de l'Atlantique est de plus en plus confrontée au problème d'hygiène et d'assainissement dû à l'absence de toilettes et de services d'assainissement adaptés (Mara et al., 2010). Les données disponibles montrent que l'accès à l'EHA reste insuffisant de façon générale au sein de cette commune. Par ailleurs, une autre étude transversale (Gomido et al., 2015) réalisée sur les facteurs sociodémographiques et comportementaux associés aux géo-helminthiases en milieu scolaire a révélé une association statistiquement significative des géohelminthiases à l'absence de lavage des mains. Ainsi, les géo-helminthiases sont trois fois plus fréquentes au sein des écoliers de cet arrondissement (Gomido et al., 2015). C'est dans ce contexte que des interventions de promotion de l'hygiène et de l'assainissement ont été mises en œuvre dans la commune de Zè. La présente étude vise à évaluer les effets des interventions du projet de Promotion de l'Hygiène et de l'Assainissement sur la santé des bénéficiaires dans la commune de Zè. Spécifiquement il s'est agit de décrire les interventions mises en œuvre dans le cadre du projet d'Hygiène et d'Assainissement ; de mesurer les effets des interventions du projet sur les Connaissances, Attitudes et Pratiques des bénéficiaires et enfin d'apprécier les effets de ces interventions sur les maladies hydro-fécales dans la commune de zè.

\section{Cadre et méthode d'études}

\section{I.1. Cadre d'étude}

La commune de Zè est située dans le département de l'Atlantique au Sud du Bénin entre $6^{\circ} 32^{\prime}$ et $6^{\circ} 87^{\prime}$ de latitude Nord et $2^{\circ} 13^{\prime}$ et $2^{\circ} 26^{\prime}$ de longitude Est. Elle s'étend sur une superficie de $653 \mathrm{~km}^{2}$ et est limitée au Nord par les communes de Zogbodomey et Toffo, au Sud par les communes d'Abomey-calavi et Tori-bossito, à l'Est par les communes de Adjohoun et Bonou, puis à l'Ouest par la commune de Allada. Cette commune compte soixante-treize (73) villages répartis sur onze (11) arrondissements Adjan, Dawé, Djigbé, Dodji Bata, Hekanmé, Koundokpoé, Sèdjè Dénou, Sèdjè houègoudo, Tangbo, Yokpo, Zè centre. Les arrondissements bénéficiaires du projet sont Sèdjè-Dénou centre, Aguiakpa, Agbohounsou et Gbozoumè et ceux non bénéficiaires que sont Agozounkpa, Domè-séko, Dawè-centre et Djigbé (figure 1). 


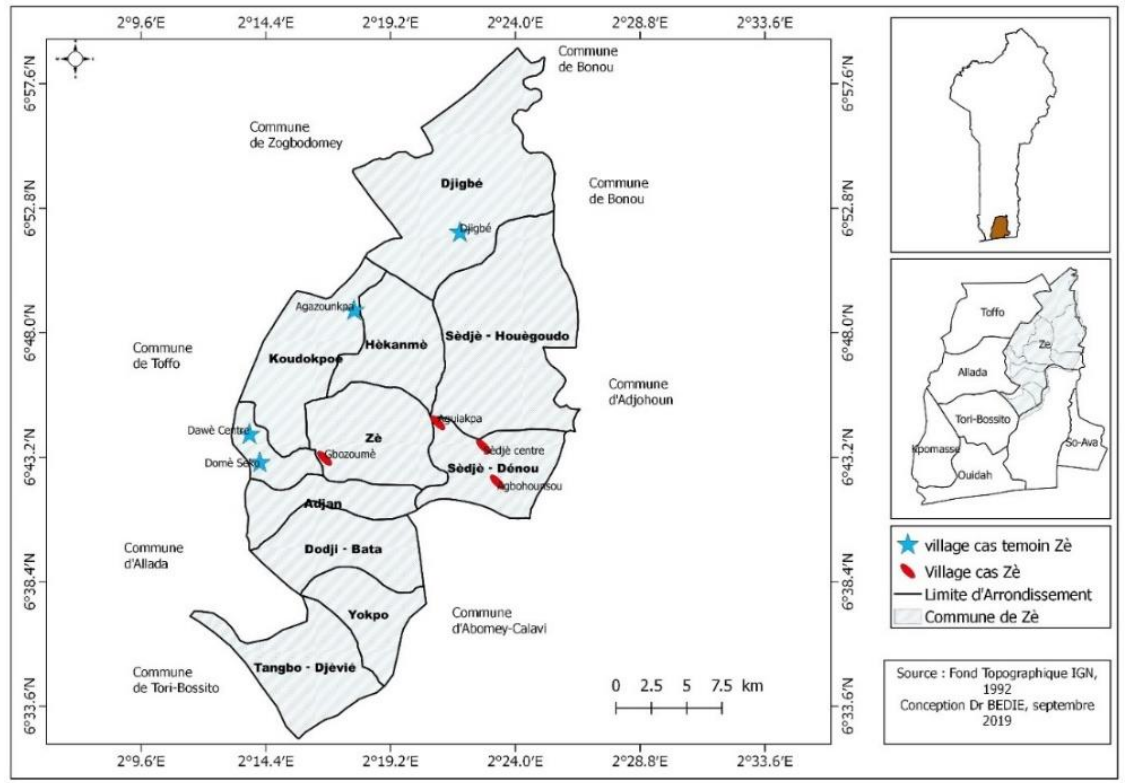

Figure 1: Situation géographique de la commune de Zè les principales sources d'eau de boisson utilisée

\section{I.2 Méthodes d'étude}

\section{I.2.1 Type d'étude}

Il s'agit d'une étude quasi-expérimentale de type ici et ailleurs portant sur l'évaluation des effets des interventions du projet de promotion de l'hygiène et de l'assainissement à Zè.

\section{I.2.2. Cibles}

La population d'étude est représentée par deux grandes cibles. La première est composée : des agents de santé ; des directeurs ou enseignants d'écoles ; des personnes ressources ; autorités politico-administratives ; responsables d'associations ou ONG de collecte des déchets. Cette première catégorie de cible est investiguée par un guide d'entretien. La deuxième catégorie est composée : des ménages (les femmes des groupements) et des écoliers. Cette deuxième catégorie est soumise à un questionnaire.

\section{I.2.3. Critères d'inclusion, critères de non inclusion et Critères d'exclusion}

Sont inclues dans l'étude les personnes qui satisfont aux conditions ciaprès : résider dans les villages des arrondissements retenus par l'étude ; donner son consentement pour répondre aux questions et/ou pour participer aux entretiens. Ne sont pas inclues dans l'étude : toutes les personnes qui n'ont pas donné leur accord pour participer à cette étude ; toutes les personnes qui étaient juste de passage dans la localité. Sont exclues de l'étude, toutes les personnes venues nouvellement ou qui sont de passage dans le secteur d'étude. 


\section{I.2.4. Echantillonnage}

Deux groupes cibles sont retenus pour servir d'échantillon. Il s'est agi des sous-groupes «ménages, les écoliers» et des «agents de santé ; enseignants d'écoles et personnes ressources». Deux types échantillonnage sont mobilisés pour les enquêtes qualitatives et quantitatives.

\subsubsection{Méthodes d'échantillonnage pour les enquêtes}

Il s'agit d'une étude mixte, à la fois qualitative et quantitative qui a été faite dans les quatre (4) arrondissements bénéficiaires du projet HA dont Sèdjè-Dénou centre, Aguiakpa, Agbohounsou et Gbozoumè. Quatre (4) autres arrondissements non bénéficiaires ont été sélectionnés dans la même commune suivant les critères d'éloignement et de caractéristiques similaires afin de mesurer les effets du projet sur les localités bénéficiaires. Il s'agit de : Agozounkpa, Domè-séko, Dawè-centre et Djigbé. Pour le choix des acteurs à interroger par une enquête ménage dans ces localités, il a été porté sur le chef de ménage et/ou son épouse. Les ménages «bénéficiaires » ont été sélectionnés de façon aléatoire à partir d'une liste des bénéficiaires de chaque localité. La stratégie du déplacement dans le sens des aiguilles d'une montre a été adoptée. La figure 1 présente la carte des sites des «bénéficiaires» et des « non bénéficiaires " retenus dans le secteur d'étude. Les quatre Ecoles Primaires Publiques: Sédjè-Dénou/A, Sédjè-Dénou/B, Aguiakpa et Agbohounsou ont été retenues. Un choix par commodité des écoliers remplissant les critères d'inclusion a été réalisé. Ainsi la taille totale de l'échantillon des écoliers est de trois cent cinquante-deux (352).

\section{I.2.4.2. Taille de l'échantillon}

- Échantillonnage pour l'enquête quantitative

Pour la réalisation de l'enquête quantitative, un recensement de tous les ménages des villages concernés par l'étude a été fait. Afin de déterminer la taille de l'échantillon, un échantillonnage aléatoire stratifié a été réalisé. Les strates sont ici représentées par les villages bénéficiaires. L'échantillon a été déterminé à l'aide de la formule de Schwartz. Un total de 322 ménages représente l'échantillon des «bénéficiaires » et selon la méthodologie adoptée (deux ménages non bénéficiaires pour un ménage bénéficiaire), la taille de l'échantillon des " non bénéficiaires » représente le double de celle de l'échantillon des "bénéficiaires », soit 644 ménages. Notifions que les localités ont été choisies en fonction de celles qui présente le plus de besoins en infrastructures EHA. La taille de l'échantillon des « non bénéficiaires » a été doublée pour avoir une fiabilité dans les résultats.

- Échantillonnage pour l'enquête qualitative

La méthode d'échantillonnage pour le volet qualitatif est le choix raisonné, cette enquête a employé un échantillon restreint. Dans ce cas, la taille 
de l'échantillon n'est pas prédéfinie avant l'enquête. La taille est arrêtée après saturation des informations au niveau des cibles. Ainsi, au terme de la collecte de données, la taille de l'échantillon du volet qualitatif a été arrêtée à 89 personnes.

\section{I.2.5. Variables}

Deux types de variables sont impliqués dans cette étude, il s'agit d'une variable dépendante et plusieurs variables indépendantes. Variable dépendante : il s'agit de l'effet du niveau d'hygiène et d'assainissement sur la santé. Variables indépendantes : Il s'agit des facteurs sociodémographiques, comportementaux et environnementaux. Caractéristiques sociodémographiques : sexe, l'âge, catégorie socioprofessionnelle, niveau scolaire, type d'habitat, ethnie. Les facteurs comportementaux liés à l'eau de boisson et les facteurs liés aux Connaissances, Attitudes et Pratiques (CAP).

\section{I.2.6. Technique et outils de collecte des données.}

Les techniques et outils de collecte utilisés pour l'étude sont: recherche documentaire, fiche de lecture ; fiche de dépouillement ; fiche de renseignements généraux sur les villages ; les méthodes de sensibilisation. La méthode aléatoire simple a été appliquée pour sélectionner trente ménages dans lesquels les prélèvements d'eau ont été faits dans chaque localité d'intervention. L'analyse microbiologique a été effectuée au laboratoire HECOTES du Centre Inter-facultaire de Formation et de Recherche en Environnement pour le Développement Durable (CIFRED). Pour la collecte des selles, des tubes plastiques munis de couvercles ont été remis aux écoliers la veille du jour des prélèvements et récupérés le lendemain matin. Trois principaux germes ont été recherchés dans ces échantillons selon la technique de Kato Katz (Ascaris lombricö̈des, Trichuris trichiura, Ankylostome duodénale) au laboratoire du centre de santé communal.

\section{I.2.7. Analyse des données}

Les logiciels Excel, SPSS 23 et Epi Info 7 ont été utilisés. Excel a permis de réaliser des tableaux, des graphiques et de générer des bases de données pour les analyses statiques avec les autres logiciel (SPSS, Epi). Le logiciel SPSS a permis le traitement des données en vue d'analyses statistiques. Epi. Info a permis de concevoir des questionnaires pour les études épidémiologiques, de saisir les réponses issues de l'enquête et d'appliquer des traitements statistiques sur ces données. 


\section{I.2.8. Aspect éthique de l'étude}

Une autorisation du déroulement de l'enquête a été obtenue auprès des autorités municipales. Tous les participants ont signé un consentement éclairé écrit. Le traitement des données a été fait dans un esprit de confidentialité.

\section{Résultats}

Les informations recueillies révèlent que toutes les parties prenantes des zones d'intervention du projet, louent les actions et évoquent fièrement les acquis : infrastructures et équipements ; comportements et pratiques ; promotion de la santé communautaire.

\section{II.1. Acquis en termes d'infrastructures et équipements du projet HA} II.1.1. La disponibilité des infrastructures de base en EHA (tableau 1)

Tableau 1. Répartition des infrastructures et équipements dans les localités bénéficiaires à zè

\begin{tabular}{|c|c|c|c|c|c|}
\hline $\begin{array}{l}\mathrm{N}^{\circ} \\
\text { d'ordre }\end{array}$ & $\begin{array}{l}\text { Infrastructures/ } \\
\text { équipements }\end{array}$ & Emplacements & $\begin{array}{l}\text { Coordon- } \\
\text { nées X }\end{array}$ & $\begin{array}{l}\text { Coordon- } \\
\text { nées } Y\end{array}$ & Localités \\
\hline 1 & Château d'eau & CS Zè centre & 421816 & 743020 & Zè centre \\
\hline 2 & Forage & EPP Aguikpa & 428072 & 745045 & \multirow[b]{2}{*}{ Aguikpa } \\
\hline 3 & WC & EPP Aguiakpa & 428104 & 745070 & \\
\hline 4 & WC ECOSAN & Sèdjè-Denou & 431232 & 743783 & \multirow{7}{*}{ Sèdjè-Denou } \\
\hline 5 & Forage & CS Sèdjè-Denou & 431255 & 743797 & \\
\hline 6 & WC ECOSAN & EPP Sèdjè-Denou B & 431407 & 743584 & \\
\hline 7 & WC ECOSAN & EPP Sèdjè-Denou A & 431320 & 743455 & \\
\hline 8 & Forage & EPP Sèdjè-Denou & 431320 & 743455 & \\
\hline 9 & Forage & Marché Sèdjè-Denou & 431577 & 743381 & \\
\hline 10 & WC ECOSAN & Marché Sèdjè-Denou & 431623 & 743392 & \\
\hline 11 & Forage1 & Agbohounssou & 431799 & 741474 & \multirow[b]{2}{*}{ Agbohounssou } \\
\hline 12 & Forage2 & Agbohounssou & 432370 & 740816 & \\
\hline 13 & Forage 2 & Gbozoumè & 430025 & 753461 & \multirow{3}{*}{ Gbozounme } \\
\hline 14 & Forage 1 & EPP Gbozounme & 429509 & 753839 & \\
\hline 15 & WC ECOSAN & Gbozounme & 429536 & 753818 & \\
\hline
\end{tabular}

Source : Travaux de terrain septembre 2019

En termes d'infrastructure, le projet a acquis au profit des Ecoles Primaires Publiques (EPP), des Centres de Santé (CS) et des communautés de la commune de Zè un total de huit (8) forages, un (1) château d'eau, et six (6) latrines ECOSAN. Ainsi, la disponibilité de point d'eau dans les villages bénéficiaires a amélioré l'accessibilité à l'eau potable dans ces localités.

\section{II.1.2. Infrastructures et équipements d'amélioration de l'accès à l'eau, l'hygiène et l'assainissement mis en place par le projet $\mathrm{HA}$ \\ Ces équipements et infrastructures sont transférés à la commune au nom des populations bénéficiaires. Cette situation leur confère une grande}


responsabilité dans l'utilisation efficace et la gestion durable de ces outils (voir photos ci-dessous).

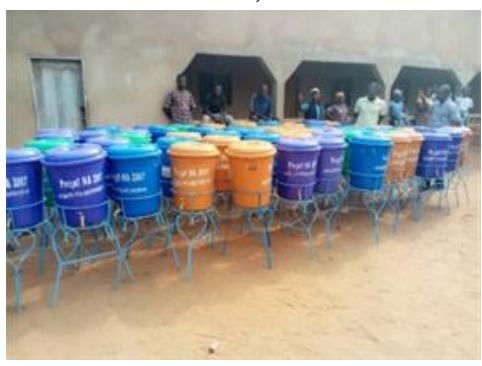

Photo 1 : Dispositif de stockage d'eau

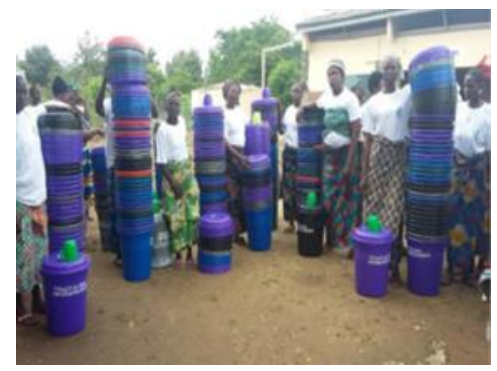

Photo 2 : Dispositifs de stockage d'eau

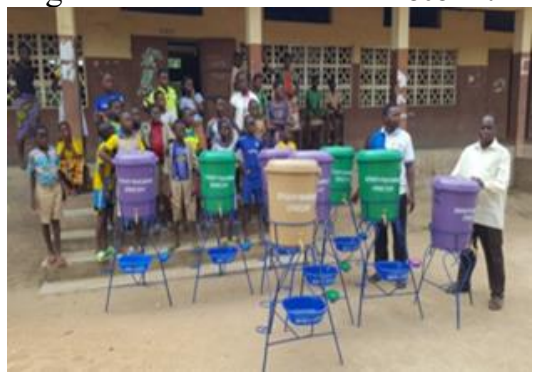

Photo 3: Matériels de lavage des mains

Source : Travaux de terrain, 2019

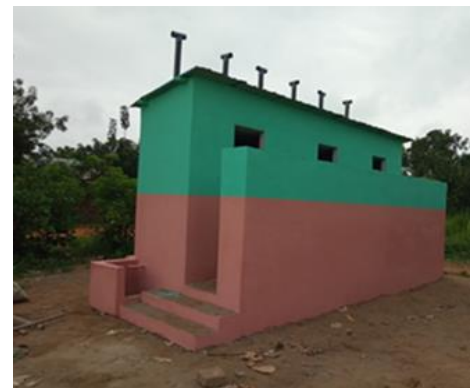

Photo 4 : Latrines ECOSAN EPP

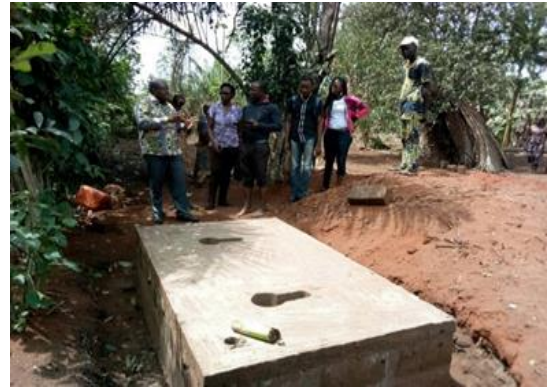

Photo 5 : Latrines domiciliaires

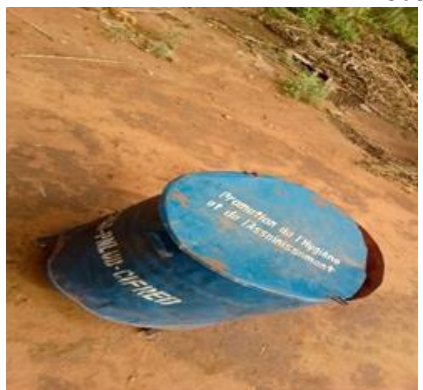

Photo 6 : Type de poubelle

Source : Travaux de terrain, 2019 


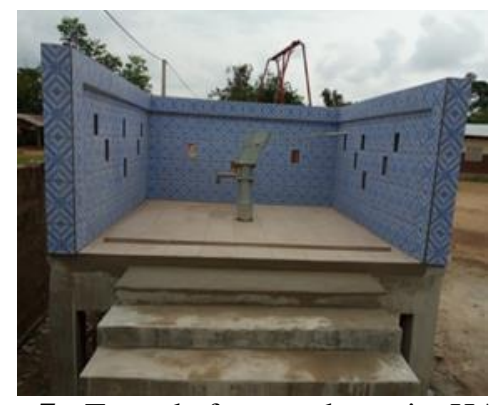

Photo 7 : Type de forages du projet HA

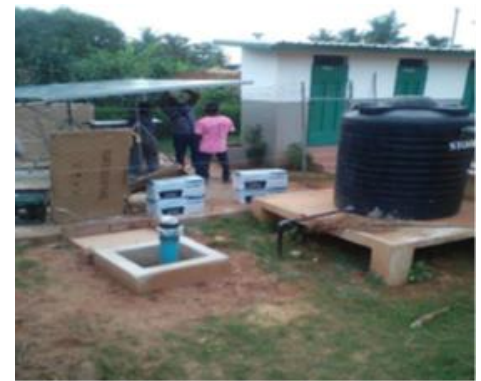

Photo 8 : Type de forages du projet HA

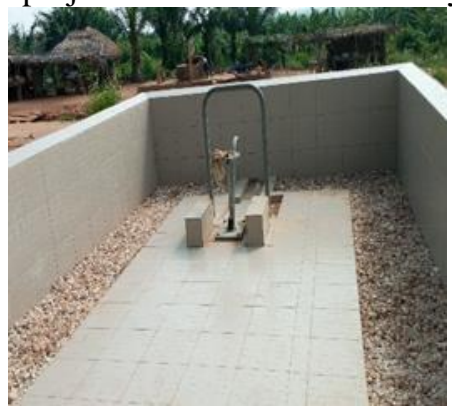

Photo 9 : Type de forages du projet HA

Source : Travaux de terrain, 2019

Le but poursuivi est l'accès équitable et durable à l'eau potable, aux infrastructures adéquates d'assainissement de base et l'appropriation des bonnes pratiques d'hygiène par les populations.

\section{II.1.3. Appréciation des résultats liés aux infrastructures par les bénéficiaires}

Deux résultats sont identifiables : la disponibilité de l'eau potable et la réduction de la distance entre les ménages et les points d'eau. Le graphique (figure 2) montre l'évolution des tendances.

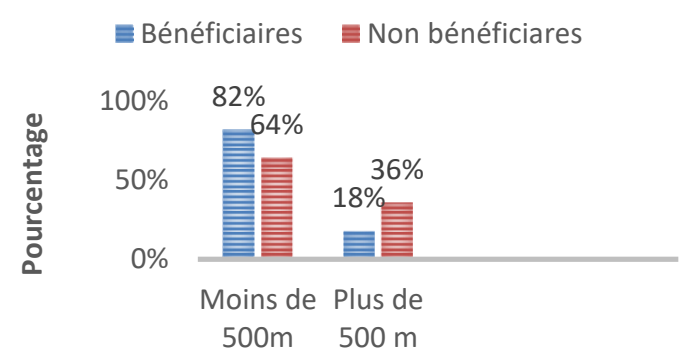

Figure 1: Répartition des ménages en fonction de la distance entre le point d'eau et les habitations

Source : Données de terrain septembre 2019 
De l'analyse de la figure 2 , il ressort que $64 \%$ des habitations des villages «bénéficiaires » sont situés à moins de 500 mètres de leur source d'eau contre $82 \%$ au niveau des villages «Non bénéficiaires ». Ainsi, la présence des forages a réduit la distance à parcourir par les femmes à la recherche d'eau potable pour le ménage ; donc moins de fatigue et gain de temps.

\section{II.2. Effet des interventions sur les Connaissances, Aptitudes et Pratiques en EHA}

\section{II.2.1 Niveau des résultats en matière de CAP liés à l'EHA}

Les méthodes de sensibilisation à savoir les projections de film et d'images sur les bonnes pratiques ont permis aux bénéficiaires d'accroître en tout premier lieu leurs connaissances sur les voies de contaminations de l'eau et celles liées aux maladies. La comparaison des deux sous-groupes d'échantillons sur les voies de contamination de l'eau de boisson, à savoir : «bénéficiaires » et «non bénéficiaires » est révélateur à ce propos.

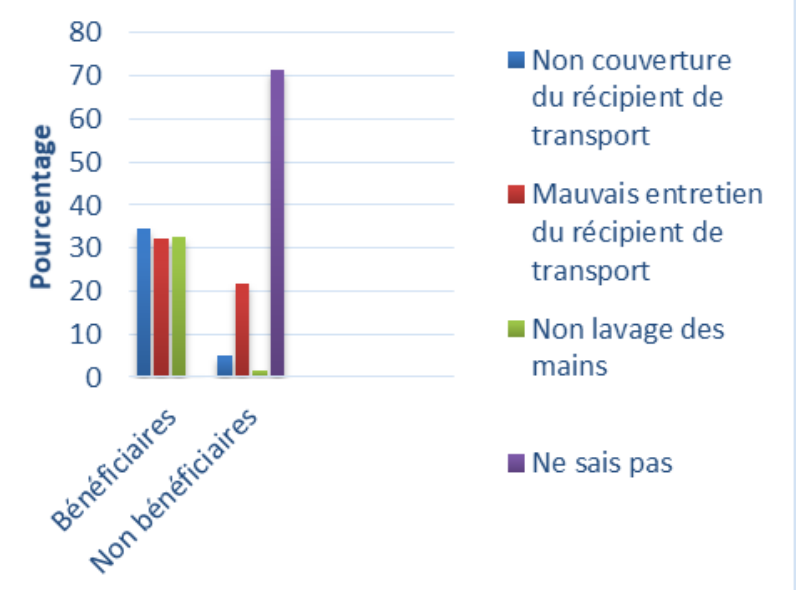

Figure 3: Niveau comparatif de connaissance sur les voies de contamination de l'eau

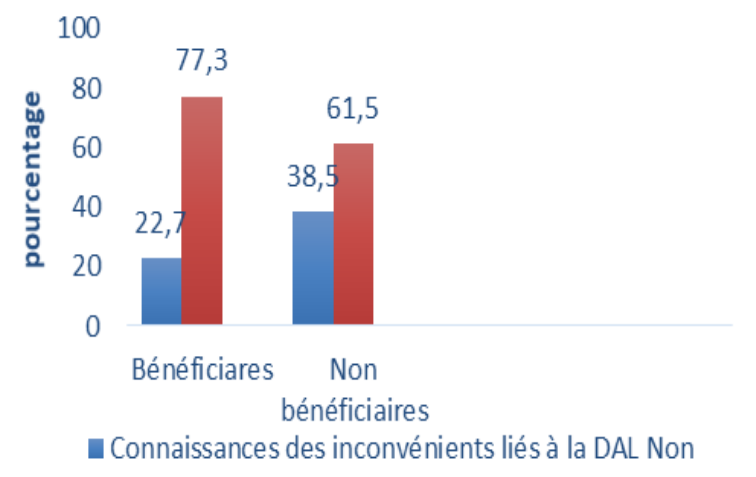

—Connaissances des inconvénients liés à la DAL Oui

Figure 4: Niveau de connaissance des inconvénients liés à la DAL 
L'analyse de la figure 3, révèle dans les localités « bénéficiaires » que les populations ont meilleure connaissance des voies de contamination de l'eau contrairement aux villages «non bénéficiaires » qui n'ont pas assez. Il en est de même pour les connaissances liées à la Défécation à l'Air Libre (DAL). En effet, de l'analyse des données relatives à la connaissance des risques liés la défécation à l'air libre (Figure 4), il ressort que 77,3\% des répondants ont connaissance contre $22,7 \%$ qui n'ont pas connaissance des inconvénients de la DAL.

\section{II.2.2. Amélioration des CAP liées à l'EHA dans les ménages bénéficiaires \\ Les figures 5 et 6 illustrent l'amélioration des CAP liées à l'EHA dans les ménages bénéficiaires.}

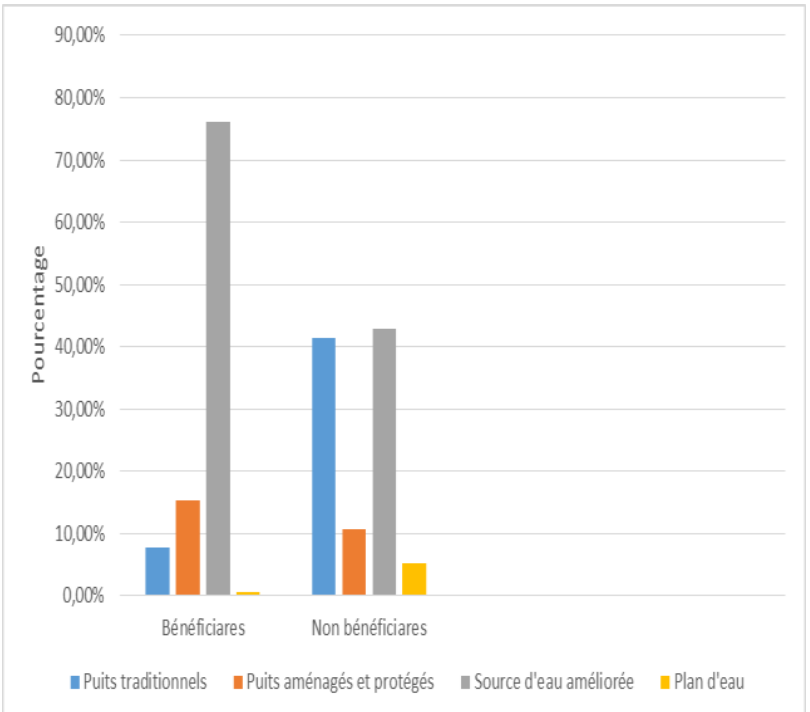

Figure 5: Répartition des ménages suivant les principales sources d'eau de boisson utilisée 


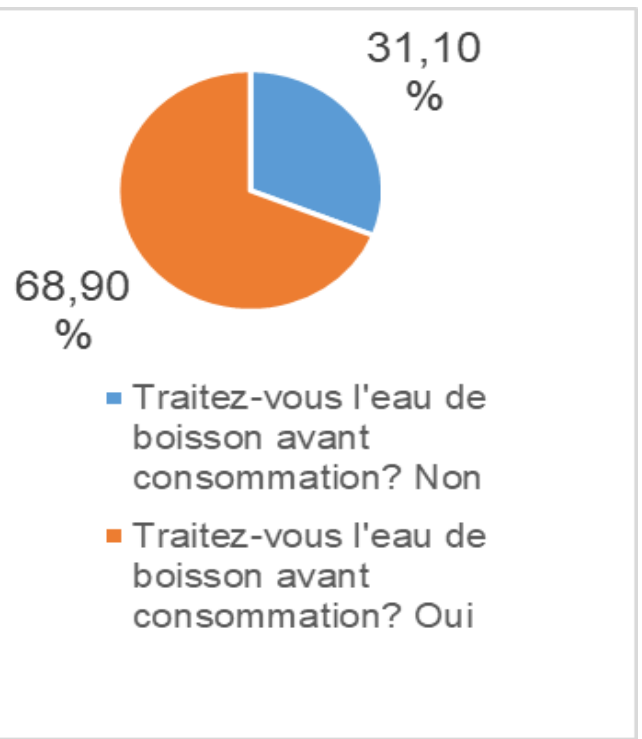

Figure 6: Traitement de l'eau avant consommation au niveau des ménages « bénéficiaires »

A travers des sensibilisations (CHAST/ PHAST) et des formations notamment par rapport à l'entretien des différentes infrastructures dont elles ont été dotées par le biais du projet HA, les populations témoignent avoir reçu des connaissances liées à l'Eau, Hygiène et Assainissement. Ces savoirs et savoir-faire restent des acquis en termes de CAP.

Quatre sources d'eau sont fréquemment utilisées par les ménages. La figure 5 montre que $76,2 \%$ des ménages utilisent les sources d'eau améliorées comme principale source d'eau de boisson contre $23,8 \%$ qui utilisent des puits traditionnels $(7,7 \%)$, des puits aménagés $(15,4 \%)$ et des plans d'eau $(0,7 \%)$. Ainsi, ces résultats montrent l'importance que revêt la mise en œuvre du projet HA dans ces localités

L'analyse de la figure 6 révèle que dans la commune de Zè, 68,9\% des ménages bénéficiaires du projet font le traitement de l'eau de boisson avant consommation contre $31,1 \%$ qui ne le font toujours pas. Le traitement de l'eau avant consommation réduit les maladies diarrhéiques. Cette pratique n'est pas utilisée par toutes les unités statistiques.

\section{II.2.3. Adoption de bonnes attitudes par les écoliers}

Les figures 7 et 8 indiquent respectivement les tendances dans les bonnes attitudes en matière d'hygiène corporel et les attitudes des écoliers en matière d'assainissement des écoles. 


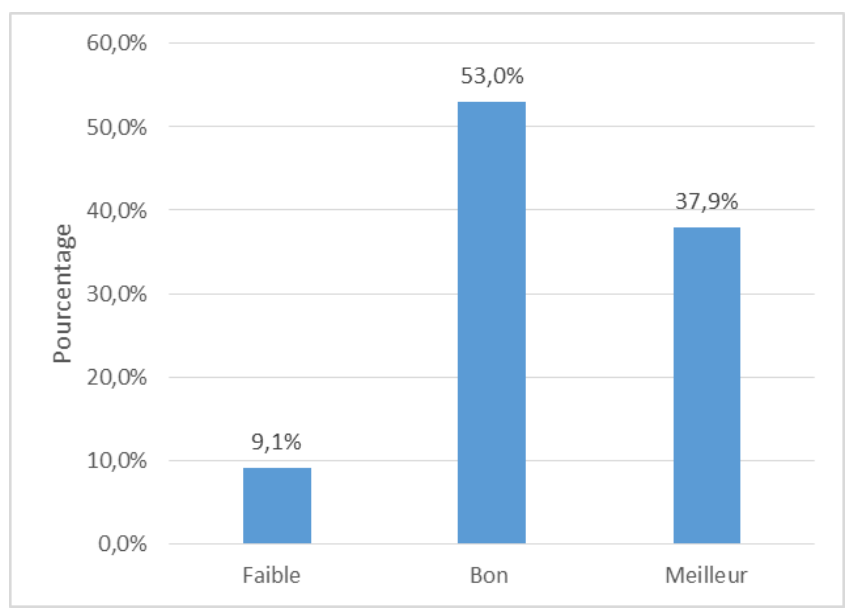

Figure 7: Niveau des attitudes des écoliers en matière d'hygiène corporelle

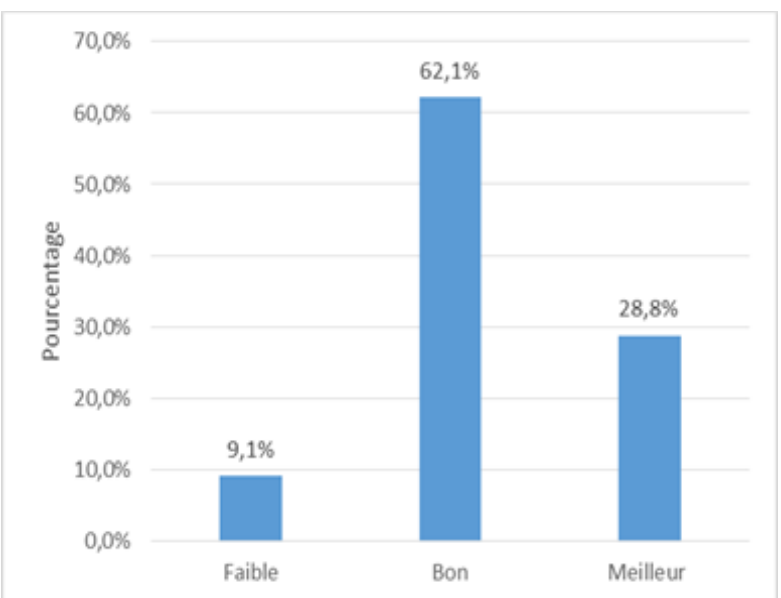

Figure 8: Niveau des attitudes des écoliers en matière d'assainissement des écoles

L'analyse de la figure 7 révèle que $90 \%$ des écoliers interviewés ont de bonne manière en matière d'hygiène corporelle. Parmi ces bonnes attitudes, on note le lavage régulier de la tenue d'école, les ongles taillés et le bain quotidien pris par les enfants. La figure 8 montre que $90 \%$ des écoliers ont adopté les bonnes pratiques et d'attitudes en matière d'assainissement de leur cadre de vie. Ces mutations en matière d'hygiène corporelle et d'assainissement du cadre de vie constatées au niveau des écoliers à travers l'adoption de meilleurs comportements sont le résultat des activités de sensibilisation de CHAST.

\section{II.3. Effet des interventions et promotion de santé}

\section{II.3.1. Niveau d'atteinte des résultats en termes de santé communautaire}

Des activités relatives aux analyses de selles et de sang, au déparasitage systématique, à la prise en charge des maladies liées à l'hygiène 
et à l'assainissement, à la potabilisation de l'eau de boisson ont permis d'infléchir les courbes des maladies diarrhéiques (figure 9).

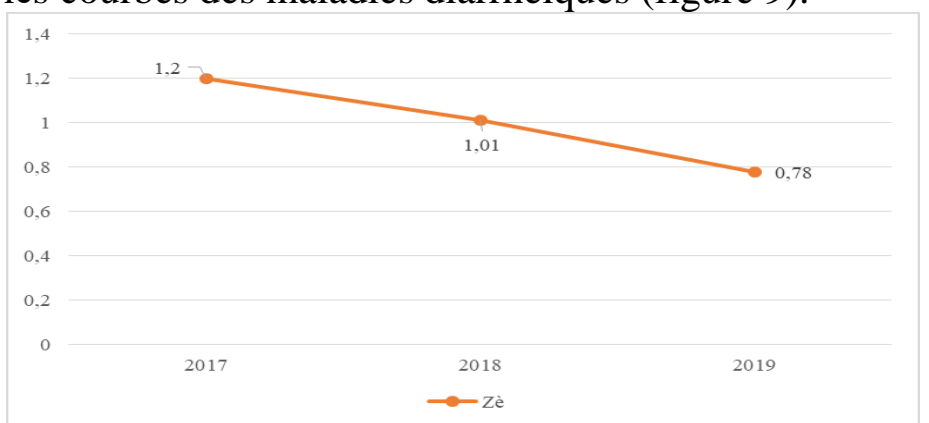

Figure 9: Évolution du Taux de morbidité des maladies hydro-fécales (\%) sur la période 2017 à 2019

La figure 9 montre l'évolution du taux de morbidité des maladies hydro-fécales sur la période allant de 2017 à 2019 dans les localités d'intervention. Sur la période, il y a eu une baisse $(1,2 \%$ à $0,78 \%)$ du taux de morbidité des maladies hydro-fécales à Zè. Cette réduction de taux morbidité est rendue possible grâce aux interventions du projet notamment à travers les communications pour un changement de comportement et la mise en pratique des notions acquises par les différentes cibles dans les zones d'intervention. De plus les écoles et les centres de santé ont été dotés des dispositifs de lavage de mains suivi de la formation à leur entretien et aux différents moments critiques auxquels il faut se laver les mains. A cela s'ajoute la formation à la préparation du savon liquide et solide. La disponibilité du savon aide désormais les écoliers, les usagers et le personnel des centres de santé dans la pratique du lavage des mains, de l'hygiène vestimentaire et corporelle.

\section{II.3.2 Réduction des taux de prévalences dans les zones d'intervention}

Les figures 10 et 11 illustrent respectivement le déparasitage régulier des ménages et la fréquence de déparasitage des enfants. 


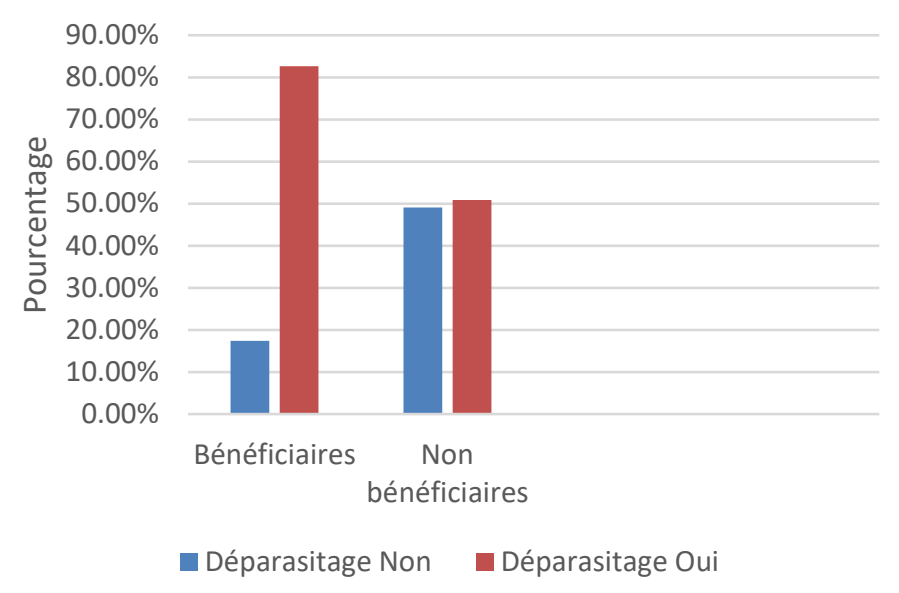

Figure 10: Répartition des ménages par commune selon la pratique du déparasitage

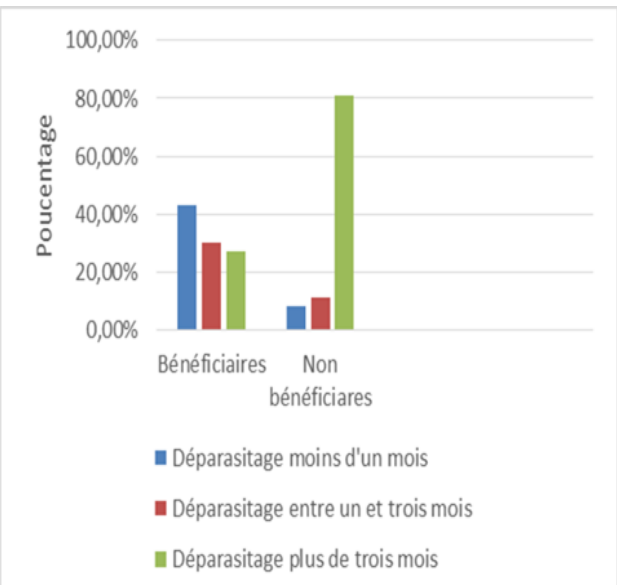

Figure 11: Répartition suivant la fréquence de déparasitage des enfants

La figure 10 montre que 82,6\% des bénéficiaires du projet dans la commune de Zè interrogés ont affirmé qu'ils se déparasitent régulièrement contre 17,4\% qui ne le font pas. En effet, 50,9\% des ménages « non bénéficiaires » interviewés se déparasitent régulièrement contre $49,1 \%$ qui ne le font pas.

La figure 11 donne les fréquences de déparasitage des enfants au niveau des bénéficiaires et non bénéficiaires de la commune. Il ressort que, $43,1 \%$ déclarent avoir déparasité leur enfant il y a moins d'un mois, $30 \%$ '’on fait il y a un à trois mois et 26,9\% se sont déparasiter il y a plus de trois mois. Ainsi, en moyenne 37,76\% des bénéficiaires du projet ont inscrit le fait de déparasiter leurs enfants dans leur habitude contre $8 \%$ au niveau des ménages non bénéficiaires du projet. Aussi, faut-il noter que la proportion des enfants déparasités des ménages «non bénéficiaires », il y a plus de trois mois $(81 \%)$ 
est largement supérieur à la moyenne de ceux qui l'ont été en moins de trois mois $(11 \%)$ dans les villages «non bénéficiaires ». Il apparaît donc un écart de $29,76 \%$ entre les pratiques relatives au déparasitage dans les villages bénéficiaires de l'intervention du projet HA et les villages non bénéficiaires. On comprend alors que les différentes sensibilisations et la mise à disposition de l'albendazole ont porté leurs fruits en inscrivant dans les pratiques habituelles des ménages bénéficiaires, l'importance de déparasiter leurs enfants.

\section{II.3.2.1. Effets de la disponibilité de latrines}

La fréquence d'utilisation des latrines par les répondants (figure 12)

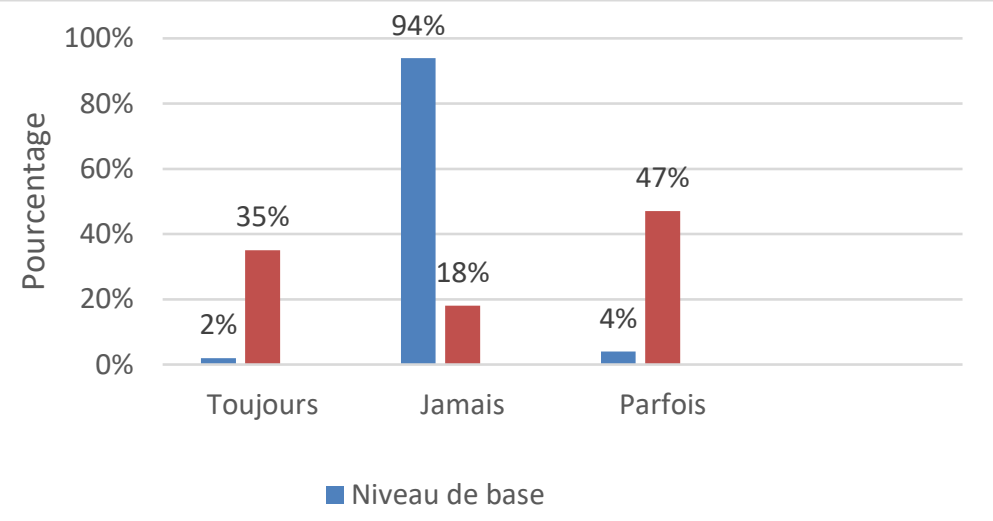

Figure 12: Fréquence d'utilisation des latrines par les répondants

Les résultats de l'étude de base sur l'utilisation des latrines construites pour les populations bénéficiaires montrent que seuls $2 \%$ des ménages utilisaient toujours ces latrines. Cependant, les résultats de l'évaluation post intervention montrent que ce taux est passé à 35\% dans cette commune. La proportion des ménages qui n'utilisent jamais de latrines est passée de $94 \%$ à $18 \%$ entre l'étude de base et l'évaluation post intervention. La défécation à l'air libre est réduite grâce à la disponibilité des latrines.

\section{II.3.2.2. Effets dans le cadre de la promotion de la santé communautaire}

Le tableau 2 montre l'effet du lavage des mains sur les maladies hydrofécales au niveau à Zè.

Tableau 2. Tableau croisé entre le lavage des mains à l'eau et au savon et l'infection hydrofécale à Zè

\begin{tabular}{llcccc}
\hline & & \multicolumn{4}{c}{ Lavage des mains avec l'eau et le savon } \\
\hline & & Jamais & Parfois & Souvent & Toujours \\
\hline \multirow{2}{*}{ Infection hydro-fécale } & Non & $4,3 \%$ & $24,4 \%$ & $52,4 \%$ & $18,9 \%$ \\
& Oui & $3,3 \%$ & $67,4 \%$ & $13,5 \%$ & $15,8 \%$ \\
\hline
\end{tabular}


Lorsqu'on recherche le lien entre le lavage des mains et la prévalence des maladies hydro-fécales, nous constatons que $67 \%$ des enquêtés à Zè, qui évoquent les maladies hydro-fécales sont celles qui ne se lavent pas régulièrement les mains à l'eau et au savon (Tableau 2). Par contre les personnes qui ne constatent plus les maladies hydro-fécales dans leurs ménages disent qu'elles se lavent souvent ou toujours les mains à l'eau et au savon. Il existe donc une forte relation entre la pratique de lavage des mains et la survenance des maladies hydro-fécales.

Tableau 3. Effet des déparasitants sur les maladies hydro-fécales

\begin{tabular}{lllllllll}
\hline & & & \multicolumn{5}{c}{ Infection hydro-fécale } \\
& & Non & \multicolumn{3}{c}{ Oui } & \multicolumn{3}{c}{ Total } \\
& & Effectif & N \% ligne & Effectif & N \% ligne & Effectif & N \% ligne \\
\hline Régularité & du & Non & 41 & $48,2 \%$ & 44 & $51,8 \%$ & 85 & $100,0 \%$ \\
déparasitage & Oui & 150 & $62,0 \%$ & 92 & $38,0 \%$ & 242 & $100,0 \%$ \\
& Total & 191 & $58,4 \%$ & 136 & $41,6 \%$ & 327 & $100,0 \%$ \\
\hline
\end{tabular}

Les résultats du tableau 3 montrent l'effet du déparasitage sur les maladies hydro-fécales au niveau des enfants. Dans les localités bénéficiaires, plus de $87 \%$ des ménages ont affirmé qu'ils donnent régulièrement des déparasitant aux enfants. Parmi ceux-ci plus de $62 \%$ déclarent que leurs enfants ne souffrent plus trop de maladies hydro-fécales. Par contre, parmi les ménages qui ne donnent pas régulièrement des déparasitants aux enfants $52 \%$ affirment que leurs enfants soufrent par moment des maladies hydro-fécales. Le déparasitage influence positivement la baisse des infections hydro fécales chez les enfants dans les localités bénéficiaires.

Tableau 4. Absence de l'administration du déparasitant sur la santé des enfants

\begin{tabular}{lllllll}
\hline $\begin{array}{l}\text { Régularité } \\
\text { déparasitage }\end{array}$ & $\mathrm{du}$ & \multicolumn{5}{c}{$\begin{array}{l}\text { Infection hydro-fécale } \\
\text { Oui }\end{array}$} \\
& $\begin{array}{l}\text { Non } \\
\text { Effectif }\end{array}$ & $\mathrm{N} \%$ ligne & Effectif & N \% ligne & $\begin{array}{l}\text { Total } \\
\text { Effectif }\end{array}$ & N \% ligne \\
\hline Non & 127 & $59,6 \%$ & 86 & $40,4 \%$ & 213 & $100,0 \%$ \\
Oui & 101 & $45,7 \%$ & 120 & $54,3 \%$ & 221 & $100,0 \%$ \\
Total & 228 & $52,5 \%$ & 206 & $47,5 \%$ & 434 & $100,0 \%$ \\
\hline
\end{tabular}

Les résultats du tableau 4 révèlent qu'environ $50 \%$ des ménages dans les localités «non bénéficiaires » n'administrent pas de déparasitant aux enfants. Parmi ces derniers plus de 54\% estiment que leurs enfants continuent de souffrir des maladies hydro-fécales. La réalisation du test de khi-deux permet de voir le lien de dépendance entre les deux variables (prise des déparasitant et prévalence des maladies hydro-fécales). Par ailleurs, la réduction des maladies liées à l'hygiène entraîne systématiquement l'épanouissement des enfants selon les répondants du monde scolaire. Pour les agents de santé, les effets positifs du projet sont entre autres, le soulagement des patients des formations sanitaires et leurs accompagnants ainsi que le 
corps médical avec la présence des latrines, une diminution des frais de santé (achat de médicaments pour les maladies diarrhéiques) ainsi que des frais pour l'approvisionner en eau satisfait.

\section{Discussion}

Les résultats révèlent la mise en place d'interventions de promotion de l'hygiène et de l'assainissement à Zè. Selon Dos Santos (2006), l'accessibilité en terme de distance aux points d'eau est un paramètre important puisqu'il détermine la quantité d'eau disponible à l'usage domestique et hygiénique. La présente étude indique que $64 \%$ des habitations des villages «bénéficiaires » sont situés à moins de 500 mètres de leur source d'eau. Ce taux est plus faible que celui obtenu par Osseni (2010) qui a montré que $100 \%$ des ménages dans les quartiers périphériques de la ville de Ouagadougou parcourent moins de $500 \mathrm{~m}$ pour aller à une borne fontaine. Cette divergence pourrait être due à la taille des échantillons, au nombre de forages et à leur répartition spatiale.

Le volet hygiène a été étudié en prenant en compte la pratique du lavage des mains. Ainsi il ressort des investigations que des latrines familiales dans la commune de Zè sont pourvues de dispositifs de lavage des mains. En effet, la présence de dispositif de lavage des mains près des latrines est un indicateur qui permet d'évaluer le taux de lavage des mains après défécation (Mooijman, 2012). Les mains favorisent la transmission des maladies du péril fécal. WSP en 2012 a conclu que les selles des enfants sont mal gérées en Afrique. Dans les Ecoles Primaires Publiques bénéficiaires, les forages et les dispositifs de lavage de mains sont fonctionnels et utilisés par les écoliers. Ces résultats corroborent ceux de Harvey et Adenya qui ont trouvé que 45\% des écoles en Zambie disposent des systèmes de lavage de mains en milieu scolaire (Harvey et Adenya, 2009).

De l'analyse de l'effet des interventions sur les Connaissances, Attitudes et Pratiques (CAP) en Eau, Hygiène et Assainissement (EHA), il ressort que les bénéficiaires ont un niveau statistiquement meilleur à celles des non bénéficiaires en matière de connaissance des sources d'eau améliorées, des voies de contamination de l'eau lors du transport et du stockage, des conséquences liées à la consommation d'une eau non potable, des conséquences liées à la défécation en plein air, des nuisances causées par une mauvaise gestion des ordures, d'utilisation de source d'eau de boisson améliorée, de traitement de l'eau de boisson, d'utilisation des récipients de stockage, de fréquence de nettoyage des récipients de stockage, de durée de conservation de l'eau de boisson et de gestion des ordures ménagères et eaux usées. Ces résultats sont semblables à ceux de l'USAID-Sénégal dans l'évaluation à mi-parcours de la performance du projet Eau et Assainissement. En effet, cette intervention dans son volet sensibilisation a permis d'améliorer la connaissance et l'utilisation des sources d'eau potables par les ménages 
bénéficiaires, la connaissance des inconvénients liés à la Défécation à l'air Libre (DAL), la diminution de la DAL, et l'utilisation des méthodes adéquates pour le traitement de l'eau de boisson (USAID, 2013).

Les différentes méthodes de sensibilisations (PHAST et CHAST) misent en œuvre chez les bénéficiaires ont permis d'accroitre le niveau de leur CAP contrairement aux non bénéficiaires. Ces résultats confirment les travaux de l'Agence d'aide à la Coopération Technique et au Développement (ACTED) qui dans son rapport d'évaluation des Connaissances, Attitudes et Pratiques (CAP) en Eau, Hygiène et Assainissement dans la commune de Mboki (République Centrafricaine) affirme que les sensibilisations en hygiène et assainissement ont permis aux bénéficiaires d'améliorer leur niveau de connaissance, d'attitude et de pratique (Nicolas, 2012). Dans le même sens, d'après l'OMS, l'initiative PHAST doit aider les communautés à améliorer les comportements en matière d'hygiène, empêcher la propagation des maladies diarrhéiques, encourager la gestion communautaire des installations d'approvisionnement en eau et d'assainissement (OMS, 1998).

Quant à la prévalence des géohelminthiases $(0,75 \%)$, elle est inférieure à la prévalence de $7,10 \%$ obtenue lors de la première étude de prévalence. Cette baisse remarquable de la prévalence obtenue au cours de cette phase comparée à la première pourrait être imputable aux interventions de promotion de l'hygiène et de l'assainissement mises en œuvre. Il s'agit notamment, de la construction de source d'approvisionnement en eau potable munies de dispositifs de lavage de mains et de contribution à l'amélioration de l'hygiène et de l'assainissement en milieu rural au Bénin: Évaluation du projet de promotion de l'hygiène et de l'assainissement dans la commune de zè savons, la construction de latrines, la sensibilisation des écoliers à l'hygiène et à l'assainissement par la méthode CHAST (Children Hygien And Sanitation Training) et le déparasitage des écoliers avec des comprimés d'Albendazole (400mg). Ces résultats sont comparable à ceux de Strunz et al. en 2014, quoi ont trouvé que l'usage d'une eau de qualité améliorée est associé à la baisse des infections aux géohelminthes (OR 0.46, IC 0.36-0.60). De même Aung Tun et al. en 2013 à Myanmar confirme suite à une campagne de déparasitage en milieu scolaire qui a fait passer le taux de prévalence de 69,7\% (étude de base) à $21 \%$ lors de l'évaluation. Aussi, d'après l'OMS, l'accès à une eau salubre et à des systèmes d'assainissement ainsi qu'une meilleure hygiène permet de réduire la morbidité imputable à l'ascaridiase de $29 \%$ et celle imputable à l'ankylostomiase de 4\% (Guerin et al., 2003). Elqaj et al. ont également conclu que pour une lutte efficace contre les parasitoses, des actions telles que l'éducation sanitaire, l'approvisionnement en eau potable, l'installation du réseau d'assainissement et surtout la sensibilisation des populations doivent être menées (Elqaj et al., 2009). 
L'évaluation de l'efficacité de l'intervention se mesure par rapport à l'atteinte des objectifs. Les populations dans les localités affectées ont acquis un savoir et un savoir-faire liés à la gestion et à l'usage de l'eau, l'hygiène et l'assainissement. Le traitement de l'eau de boisson, le stockage et même certaines localités qui ne disposaient pas d'un point d'eau potable ont obtenu un forage. Les populations ont été sensibilisées par rapport aux divers usages de l'eau. Les écoliers et les femmes sont les premiers concernés par les interventions du projet $\mathrm{HA}$, dans les zones d'interventions où ils jouent un rôle prépondérant dans l'approvisionnement en eau de la famille, le maintien des conditions d'hygiène et de salubrité. Prendre en compte cette dimension relève de l'intégration du genre dans la mise en œuvre du projet HA. Cependant, la stratégie WASH vise les populations vulnérables au sens communautaire au sein desquelles il y a plusieurs catégories de groupes vulnérables au sens individuel (personnes indigentes, malades mentaux, handicapés physiques, personnes âgées, etc.). A travers l'analyse des résultats, nous avons constaté que ledit projet semble ne pas avoir orienté de façon spécifique ses actions vers eux. Il n'y a d'ailleurs aucune mention à ce sujet ni à la mise en œuvre, ni à l'exploitation des infrastructures. Au regard de ce qui précède, les dimensions genre et équité ont été faiblement intégrées dans la conception et dans la mise en œuvre des interventions du projet HA.

\section{Conclusion}

L'insuffisance d'ouvrages et d'équipements sanitaires appropriés ; la mauvaise évacuation des eaux pluviales et usées ; la mauvaise gestion des différents déchets ; la défécation à l'air libre et les comportements, attitudes et pratiques des populations inadaptées en matière d'hygiène et d'assainissement sont des problèmes auxquels sont confrontés la population de la commune de Zè. La mise en œuvre du projet HA dans à Zè a permis de constater que dans les localités cibles de la commune de Zè, les taux de personnes ayant de bonnes pratiques d'hygiène et d'assainissement est passé de $31,01 \%$ à $73,08 \%$. Ces résultats laissent des effets et des impacts certains sur la santé des communautés cibles et leur autonomisation. C'est pourquoi, les taux de morbidité liée aux maladies hydro-fécales sont passés de 1,2\%o à 0,78\%. Dans le cadre de la pérennisation des acquis, le soin est laissé aux associations et groupements de femmes qui se sont engagés grâce au projet HA dans l'amélioration de leurs conditions d'hygiène et d'assainissement.

\section{References:}

1. Awofeso N, Kwa B, Peckham S. Water, sanitation, and public health. 2013. Journal of environmental and public health, ID 641749, 2. http://dx.doi.org/10.1155/2013/641749. 
2. Bartram J, Cairncross S. Hygiene, sanitation, and water: forgotten foundations of health. 2010. PLoS medicine 7(11). https://doi.org/10.1371/journal.pmed.1000367.

3. Campbell AH, Marzinelli EM, Vergés A, Coleman MA, Steinberg PD. Towards Restoration of Missing Underwater Forests. 2014. PLoS ONE 9(1).

4. Elqaj M, Belghyti D, Ahami A, Loutfi H, Elkharrim K, Taboz Y. Prévalence des parasitoses intestinales chez les écoliers en milieu rural Kenitra - Maroc. 2009. World Journal of Biological Research, 2: 1-6.

5. Guerin PG, Cordier S, Viau C, Quenel P, Dewailly E. Environnement et santé publique : fondement et pratiques. 2003.1062 p.

6. Harvey PA, Adenya EA. Water, sanitation and hygiene: sustainable development and multisectoral approaches. 2009. An assessment of sanitation and hygiene in primary schools in Zambia. $8 \mathrm{p}$.

7. INSAE (2019). Enquête Démographique et de Santé au Bénin, 20172018, Cotonou, Bénin et Rockville, Maryland, USA : INSAE et ICF, 675p.

8. Mara D, Lane J, Scott B, Trouba D. Sanitation and health. 2010. PLoS medicine 7(11).

https://www.ncbi.nlm.nih.gov/pmc/articles/PMC2981586/

9. 201202_Mboki_EHA_ACTED-public.pdf [Internet]. [cité 17 avr 2020]. Disponible sur :

https://www.humanitarianresponse.info/sites/www.humanitarianresp onse.info/files/201202_Mboki_EHA_ACTED-public.pdf.

10. McGinnis SM, McKeon T, Desai R, Ejelonu A, Laskowski S, Murphy HM. A systematic review: costing and financing of water, sanitation, and hygiene (WASH) in schools. 2017. International journal of environmental research and public health, 14 (4): 442. https://doi.org/10.3390/ijerph14040442.

11. Mooijman A. Eau, Assainissement et Hygiène (WASH) dans les écoles : un complément au manuel des écoles amies des enfants. 2012. UNICEF, 58p.

12. Nicolas RM. Evaluation des Connaissances Attitudes et Pratiques en EHA. Commune de Mboki. 2012. ACTED, 24 p.

13. Ogden S, Gallo K, Davis S, Addiss D, Haddad D. WASH et les maladies tropicales négligées (manuel à l'intention des responsables de la mise en œuvre du programme wash, Atlanta, États-Unis). 2013. $58 \mathrm{p}$.

14. https://www.pseau.org/outils/ouvrages/cww_wash_et_les_maladies_t ropicales_negligees_2013.pdf 
15. OMS. Manuel pas à pas sur PHAST: une approche participative pour enrayer les maladies diarréhiques. OMS Genève, 1998. OMS : $\mathrm{WHO} / \mathrm{EOS} / 98.3,135 \mathrm{p}$.

16. OMS. Liens entre l'eau, l'assainissement, l'hygiène et la santé faits. Genève. 2004 https://apps.who.int/iris/handle/10665/69490.

17. OMS. Agir pour réduire l'impact mondial des maladies tropicales négligées : premier rapport de l'OMS sur les maladies tropicales négligées. 2011. ISBN 9789242564099, 172p. https://apps.who.int/iris/handle/10665/44694.

18. OMS. Liste mondiale de référence des 100 indicateurs sanitaires de base. Organisation Mondiale de la Santé. 2015. WHO/HIS/HSI/2015.3, 139 p.

https://apps.who.int/iris/handle/10665/204687.

ttps://www.who.int/water_sanitation_health/publications/drinkingwater-quality-gui.

19. OMS. Directives de qualité pour l'eau de boisson. 4e éd. intégrant le premier additif. 2017. ISBN: 978-92-4-254995-9, $564 \mathrm{p}$.

20. Santos, S., 2006. Accès à l'eau et enjeu socio-sanitaire à Ouagadougou, Espace, population, société2-3 pp 271-285.

21. Strunz EC, Addiss DG, Stocks ME, Ogden S, Utzinger J, Freeman MC. Water, sanitation, hygiene, and soil-transmitted helminth infection: a systematic review and meta-analysis. PLoS medicine, 2014. 11(3).

https://www.ncbi.nlm.nih.gov/pmc/articles/PMC3965411.

22. Tun A, Myat SM, Gabrielli AF, Montresor A. Control of soiltransmitted helminthiasis in Myanmar: results of 7 years of deworming. 2013. Tropical Medicine and International Health, 18 (8): 1017-1020. https://europepmc.org/article/med/23701018.

23. USAID-Senegal. Evaluation à mi-parcours de la performance du projet Eau et Assainissement de l'USAID Sénégal. USAID, 2013.179 p.

24. WSP. Impacts économiques d'un mauvais assainissement en Afrique. 2012. 6p.

https://www.pseau.org/outils/biblio/resume.php?d=3640\&l=fr 